\title{
Low-to-Medium-Frequency AC Impedance Spectroscopy Investigations of Nanocrystalline Calcium Silicate Hydrate Dried Powders
}

\author{
Fabrice Leroux, ${ }^{\ddagger, \S}$ Julie Russias, ${ }^{\bullet}$ Celine Cau-dit-Coumes, ${ }^{\bullet}$ Fabien Frizon, ${ }^{\natural}$ and Guillaume Renaudin ${ }^{\dagger, \S, \|}$ \\ ${ }^{\ddagger}$ Laboratoire des Matériaux Inorganiques, Clermont Université, Université Blaise Pascal 10448, \\ F-63000 Clermont-Ferrand, France \\ ${ }^{\S}$ CNRS, UMR 6002, LMI, F-63177 Aubière, France \\ `Commissariat à l'Energie Atomique et aux Energies Alternatives, DEN, Marcoule, Waste Treatment and \\ Conditionning Research Department, F-30207 Bagnols sur Cèze, France
}

"ENSCCF, Laboratoire des Matériaux Inorganiques, Clermont Université, Blaise Pascal 10448, F-63000 Clermont-Ferrand, France

\begin{abstract}
Low-to-medium-frequency range impedance spectroscopy was used to investigate two series of dried calcium silicate hydrates with or without aluminum atoms, $\mathrm{C}-\mathrm{S}-\mathrm{H}$ and $\mathrm{C}-\mathrm{A}-\mathrm{S}-\mathrm{H}$. Over four decades in frequency, sample Nyquist plots were fitted by adopting an equivalent circuit using constant phase elements (CPE). Conductivity values of the order of $10^{-9}-10^{-10} \mathrm{~S} / \mathrm{cm}$ were obtained at $316 \mathrm{~K}$. The presence of $\mathrm{CPE}$ characteristic of the depleted semicircle at high frequency was related to a fractal dimension ranging from 2.4 up to 2.7. Above $316 \mathrm{~K}$, the impedance spectra behaved unpredictably due to the dehydration process, while below $316 \mathrm{~K}$ the behavior was followed by adopting the modulus loss factor. The associated peak maximum variation is of the Arrhenius-type. The entire behavior may be interpreted by ionic motion and charge accumulation in addition to dielectric polarization at the grain boundaries associated to low fractal surface.
\end{abstract}

\section{Introduction}

$\mathrm{T}$ HE insertion of $\mathrm{Al}$ atoms in the $\mathrm{C}-\mathrm{S}-\mathrm{H}$ phase (calcium silicate hydrate), the main component of Portland cement hydrated paste responsible for cohesion properties and durability, has been largely studied in the past. ${ }^{1-8}$ Additionally for cementbased materials, permeability is closely related to durability, low permeability being identified with improved durability. To characterize such properties as well as to unravel middle range order structure such as grain boundaries, electrochemical impedance spectroscopy (EIS) is a suitable noninvasive and non destructive characterization based on the measure of the conductivity of migrating charge carriers in an applied electric field. The amount of water and its interaction with $\mathrm{C}-\mathrm{S}-\mathrm{H}$ environment affect the dielectric response (charge carriers and dipolar species). ${ }^{9,10}$ Indeed, electrical conductivity versus time (so called conductograms) is considered as the fingerprint of specific formulations and cure conditions as well as being used to control the electrical properties of cementitious binder for the cathodic protection of reinforced concrete structure. ${ }^{11}$ EIS was also used to study the effect of silica fume on the evolution of the microstructure on densified cements, ${ }^{12,13}$ the effect of accelerators (calcium chloride, sodium

D. Viehland - contributing editor

Manuscript No. 28564. Received September 10, 2010; approved January 7, 2011.

Author to whom correspondence should be addressed. e-mail: guillaume.renaudin@ ensccf.fr formate) and of retarders (citric acid), ${ }^{14}$ and of admixtures such as acrylic polyelectrolyte, ${ }^{15}$ calcium naphthalene-sulfonates ${ }^{16,17}$ as well as the study of crack propagation and fiber orientation. ${ }^{18}$

From a structural point of view, the cohesion of cement paste is due to the formation of a network of nanoparticles of $\mathrm{C}-\mathrm{S}-\mathrm{H} .{ }^{19}$ By using a dielectric continuum model, these authors have shown that at high surface charge densities, the surface charge of $\mathrm{C}-\mathrm{S}-\mathrm{H}$ is overcompensated by $\mathrm{Ca}^{2+}$ ions, giving a reversal of the apparent particle charge and that a correlation of closely located ions causes an attraction between the particle surfaces. Such cohesion as well as the gain in strength in the early age of the cement paste results from the interaction between $\mathrm{C}-\mathrm{S}-\mathrm{H}$ particles and the changes in ionic concentration in the interstitial solution. ${ }^{20-23}$ In cement paste, additional polarization may also arise from the electrode-fluid junction and therefore limits the diffusive motion of the carriers. ${ }^{24}$ The highfrequency region was particularly studied to correlate the electrical response of the concrete to physical characteristics of its microstructure ${ }^{25-27}$ and very high values of the dielectric constant beyond expectations were explained on the basis of a dielectric amplification factor (DAF) mechanism. ${ }^{25}$ Misinterpretation of the high-frequency loop has indeed given rise to dielectric constants $>10^{3}$; these dielectric constant values were first explained by the DAF factor, ${ }^{28}$ but later on rather explained by two concomitant processes: one due to the solid phase and the second one due to the ionic motion of free ions in the electrolyte drilling the pores. ${ }^{29,30}$ One should report the study comparing porous silico-aluminate ceramic to aluminous cement in order to disentangle the dielectric behavior of hardened cementitious material ${ }^{31}$ and the use of reflectometry to permit to investigate the behavior in high frequency from $10^{3}$ to $10^{9} \mathrm{~Hz}$, well above a conventional impedance analyzer. ${ }^{32}$

We investigate here the effect of the change in the chemical composition for both series $\mathrm{C}-\mathrm{S}-\mathrm{H}$ and $\mathrm{C}-\mathrm{A}-\mathrm{S}-\mathrm{H}$ by EIS in the frequency domain $0.1 \mathrm{~Hz}$ to $1 \mathrm{kHz}$. In two companion papers, we have recently reinvestigated the long range order by $\mathrm{XRD}^{33}$ as well as the local order by NMR and Raman spectroscopy. ${ }^{34}$ Indeed, previous Rietveld analyses performed on both series have clearly shown that (1) a unique structural model allowed to describe the $\mathrm{C}-\mathrm{S}-\mathrm{H}$ structure whatever the $\mathrm{C} / \mathrm{S}$ ratio and (2) the insertion of aluminum into the $\mathrm{C}-\mathrm{S}-\mathrm{H}$ structure led to a deterioration of the crystallinity and to a systematic increase of the basal spacing of about $2 \AA$ whatever the $\mathrm{Ca} /(\mathrm{Si}+\mathrm{Al})$ ratio (at constant $\mathrm{Al} / \mathrm{Si}$ ratio). The present paper investigates the impact of such local and long range structural changes on the conductive and dielectric properties. One should observe the paucity of information in 
the literature concerning the use of AC impedance spectroscopy to characterize dry cement-type material. Indeed, study on dry cement-related phases is scarcely reported, whereas a lot of attention is still being paid on the physical origin of the microstructure on fresh cement pastes, ${ }^{35,36}$ this to better understand the chemical, physical and mechanical processes that turn water-cement mixes into stone-like solids.

\section{Experimental Procedure}

\section{(1) $C-S-H$ and $C-A-S-H$ Syntheses}

Syntheses of $\mathrm{C}-\mathrm{S}-\mathrm{H}$ and Na-free $\mathrm{C}-\mathrm{A}-\mathrm{S}-\mathrm{H}$ samples were described in the first part of the study. ${ }^{33}$ The samples studied here were designed by $\mathrm{C}-\mathrm{S}-\mathrm{H}_{-} n$ and $\mathrm{C}-\mathrm{A}-\mathrm{S}-\mathrm{H}_{-} n$, with $n=$ $\mathrm{Ca} / \mathrm{Si}=0.8,1.0,1.1$, and $1 . \overline{3}$ for respectively the Al-free and Al-containing syntheses.

C-S-H $n$ series was synthesized from silica (Aerosil 380, Degussa, $\bar{P}$ aris, France) and calcium oxide ( $99.9 \%$ pure, Aldrich, Lyon, France) freshly decarbonated for $3 \mathrm{~h}$ at $900^{\circ} \mathrm{C}$. Demineralized and decarbonated water was added to reach a water/solid ratio of 50 .

$\mathrm{C}-\mathrm{A}-\mathrm{S}-\mathrm{H} \_n$ series, with a nominal $\mathrm{Al} / \mathrm{Si}$ atomic ratio of 0.1 , was synthesized from silica (Aerosil 380, Degussa) and calcium oxide (99.9\% pure, Aldrich) freshly decarbonated for $3 \mathrm{~h}$ at $900^{\circ} \mathrm{C}$ incorporated in a saturated solution of tricalcium aluminate $\left(\mathrm{C}_{3} \mathrm{~A} \quad 1 \mathrm{~g} / \mathrm{L}\right.$ in demineralized and decarbonated water). Calcium and aluminum concentrations in the solution were determined by ICP-AES; respectively 210.0 and $114.4 \mathrm{mg} / \mathrm{L}$. Silica and calcium oxide were incorporated in these filtered solutions (at $0.45 \mu \mathrm{m}$ ) to reach a water/solid ratio of 50 , a theoretical $\mathrm{Al} / \mathrm{Si}$ atomic ratio of 0.1 , and the five $\mathrm{Ca} /(\mathrm{Al}+\mathrm{Si})$ ratios from 0.8 to 1.5 .

All the suspensions, from both $\mathrm{C}-\mathrm{S}-\mathrm{H}$ and $\mathrm{C}-\mathrm{A}-\mathrm{S}-\mathrm{H}$ syntheses, were stored at $20^{\circ} \mathrm{C}$ under $\mathrm{N}_{2}$ atmosphere for three weeks under stirring in closed polypropylene bottles. They were then filtered under nitrogen and rinsed with acetone. The precipitates were subsequently dried in a desiccator, under slight vacuum $(\approx 0.7 \mathrm{~atm})$, over silica gel, at room temperature. Samples were left over 6 months at an RH of $50 \%$ to reach hydration equilibrium. Sample compositions determined in Renaudin et al. $^{33}$ are indicated in Table I.

\section{(2) Analytical Technique}

(A) Technique and Equipment: EIS measurements were performed on pressed sample pellet with a two-Pt contacting electrode in the form of a plain capacitor (13 $\mathrm{mm}$ diameter). The cell was directly wired to an analyzer. Measurements were performed on a temperature domain ranging from 253 to $341 \mathrm{~K}$ in the frequency interval $0.1 \mathrm{~Hz}$ to $1 \mathrm{kHz}$.

(B) Data Processing: The impedance and admittance data were fitted using either the impedance or admittance functions as defined by adopting equivalent circuit of $R_{\mathrm{i}}$ and $C_{\mathrm{i}} / n_{\mathrm{i}}$ constant phase element (CPE). A minimum number of time constants $R_{\mathrm{i}} C_{\mathrm{i}}$ was used to reproduce the $Z^{\prime}+j Z^{\prime \prime}$ spectra, i.e. the real and imaginary part of the experimental impedance $Z$ at a given temperature. In a first approximation, a parallel $/ R \mathrm{CPE}_{\mathrm{p}}$ element was used to reproduce the high-frequency profile while a series $+R C C_{\mathrm{s}}$ was considered for the low-frequency domain. Such equivalent circuit was adopted because it may be compared with previous data. ${ }^{24}$ The experimental data points obtained in both series were simulated using nonlinear least-squares fits. The errors associated with the determined parameters were within $\pm 5 \%$.

Electrical relaxation phenomena were analyzed through different formalisms. However, by adopting the electric modulus formalism for the interpretation of bulk relaxation properties, some advantages upon other treatments were observed, as the conductivity relaxation is becoming prominent due to the suppression of the electrode effects. . $^{37,38}$

Conductivity and dielectric constant were obtained by converting the resistance and capacitance from the impedance

Table I. Experimental Data and Refinement Results of the EI Spectra at $316 \mathrm{~K}$ Adopting a Three-Element Model $+\mathrm{CPE}_{\mathrm{s}} / \mathrm{CPE}_{\mathrm{p}} \boldsymbol{R}$

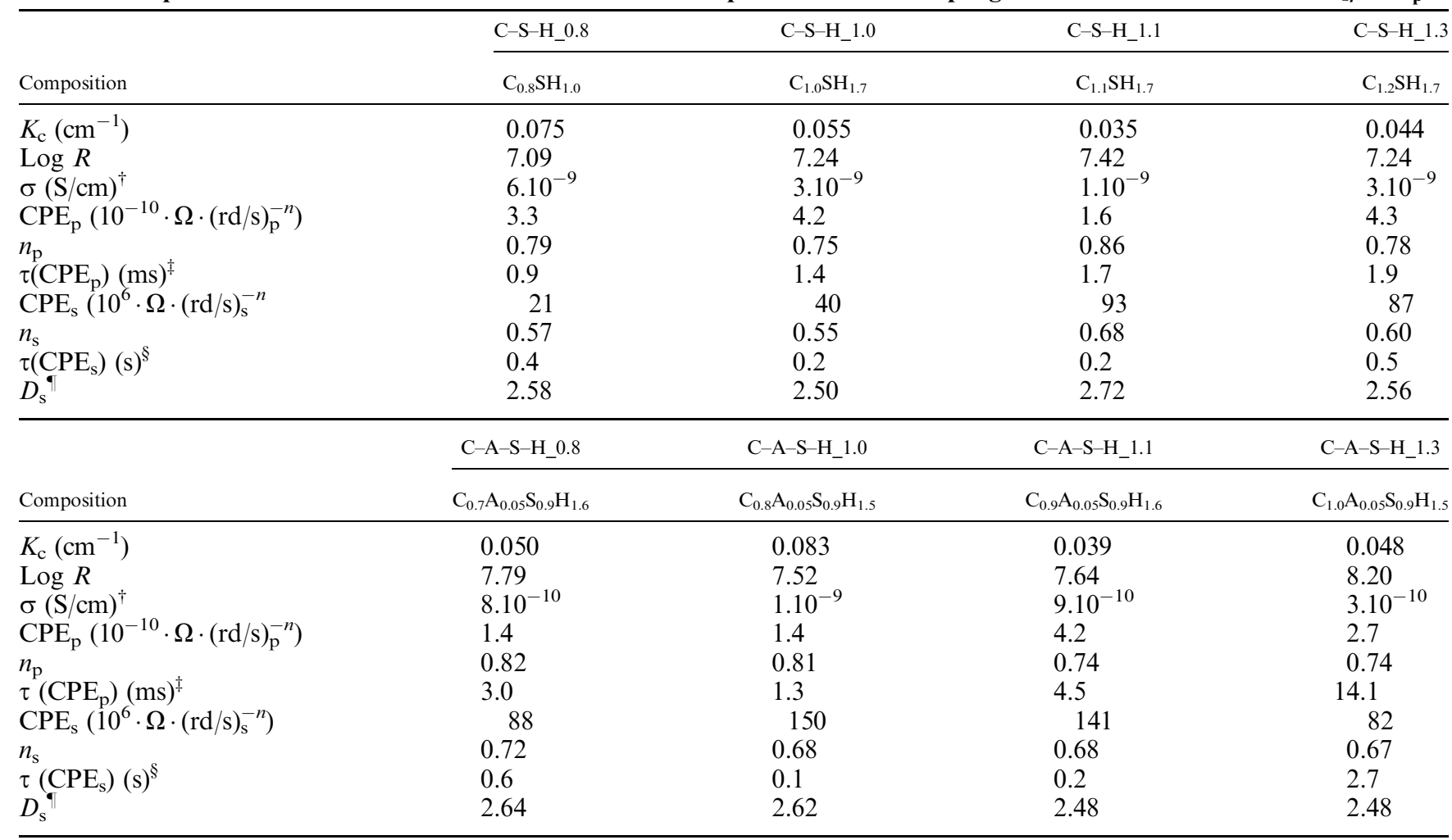

$K_{\mathrm{c}}, \sigma$, and $D_{\mathrm{s}}$ are geometric cell constant, the conductivity, and the fractal interface number.

${ }^{\dagger} \sigma(\mathrm{S} / \mathrm{cm})=K_{\mathrm{c}} / R \cdot{ }^{\ddagger} \tau\left(\mathrm{CPE}_{\mathrm{p}}\right)=\left(R \mathrm{CPE}_{\mathrm{p}}\right)_{\mathrm{p}}^{1 / n} \cdot{ }^{\S} \tau\left(\mathrm{CPE}_{\mathrm{p}}\right)=\left(R / \mathrm{CPE}_{\mathrm{s}}\right)_{\mathrm{s}}^{1 / n} \cdot{ }^{\bullet} n_{\mathrm{p}}=\left(D_{\mathrm{s}}-1\right) / 2$. 
data and plotted in the frequency domain. Electric modulus is then defined as the inverse quantity of the complex permittivity by:

$$
\begin{aligned}
& M^{*}=M^{\prime}+j M^{\prime \prime}=1 / \varepsilon^{*}=j \omega Z^{*}\left(j^{2}=-1\right) \\
& M^{\prime}=-\omega Z^{\prime \prime} \\
& M^{\prime \prime}=\omega Z^{\prime}
\end{aligned}
$$

where $M^{\prime}$ is the real and $M^{\prime \prime}$ the imaginary part of the electric modulus, respectively, and $\varepsilon^{*}, Z^{*}$, and $\omega$ are the complex permittivity, complex impedance, and the pulsation, respectively.

\section{Discussion}

\section{(1) Conduction Study}

Typical Nyquist plots of the complex impedance data of C-S-H_0.8 are displayed in Fig. 1. The impedance plots are dependent on the temperature of measurement. At a temperature lower than $286 \mathrm{~K}$, the impedance plots show a large semicircle over the frequency range (Fig. 1(a)), while at higher temperature an inclined and individual straight line is observed in the lower frequency part in addition to one depressed semicircular arc in the mid to higher frequency range (Fig. 1(b)). Indeed, at low temperature, the features are not prominent, whereas raising the temperature gives a typical two-arc response with the electrode polarization arc
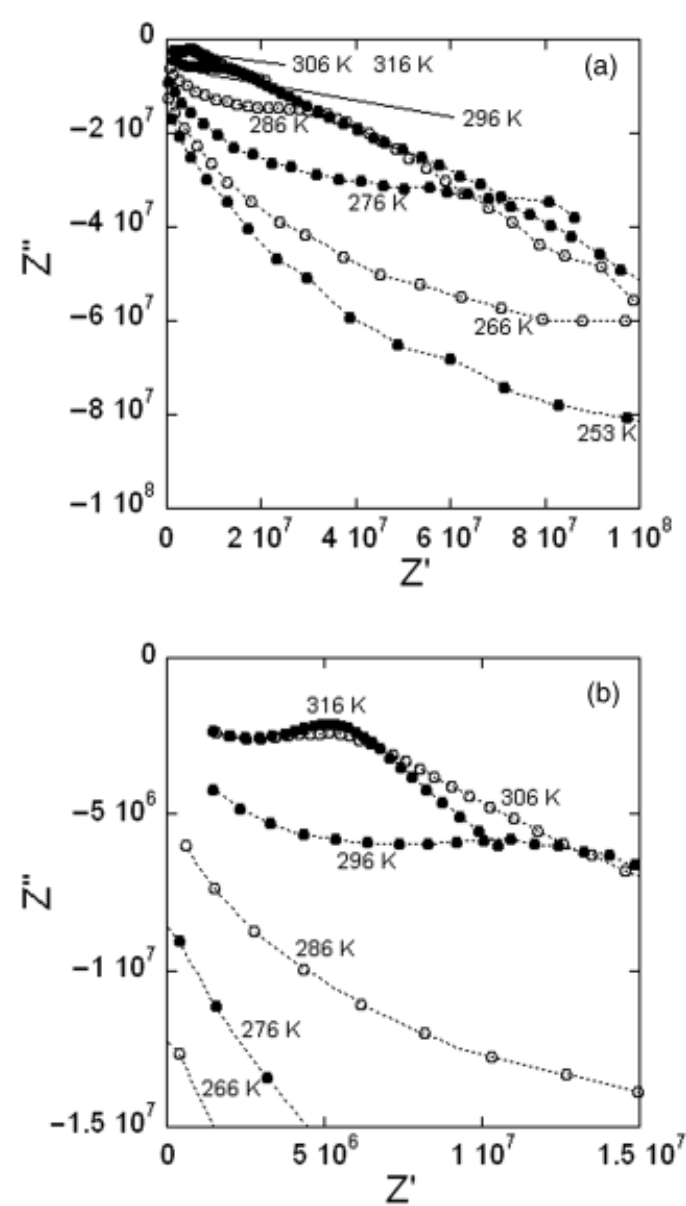

Fig. 1. Nyquist plot of the electrochemical impedance spectra of C-S-H_0.8 at indicated temperatures in (a) the entire frequency domain and (b) the high-frequency part corresponding to the lower impedance response. forming the right-hand side of a V-shaped plot and with the bulk arc forming the left-hand side (Fig. 1(b)). The bulk semicircular arc is not fully developed due to the upper frequency limit of $1 \mathrm{kHz}$.

The semicircle appearing at high to middle frequency is associated with the bulk resistance and a nonideal capacitor $\mathrm{CPE}_{\mathrm{p}}$ in parallel while the response in the lower frequency domain is characteristic of interfacial resistance in the series with the double-layer capacitance $\mathrm{CPE}_{\mathrm{s}}$. Nonideal capacitor is usually known as a CPE, which accounts for the observed depression of semicircles.

When present, the intercept or its projection on the real axis corresponds to the sample bulk resistance $R$ of the system. $R$ usually obtained at the cusp between the electrode and bulk arcs here is refined using the entire frequency domain, together with $\mathrm{CPE}_{\mathrm{p}}$ and $\mathrm{CPE}_{\mathrm{s}}$. The resulting resistances are used to determine the conductivity.

Figure 2 shows the quality of the refinement for both series at $316 \mathrm{~K}$, and $R, \mathrm{CPE}_{\mathrm{p}}$ and $\mathrm{CPE}_{\mathrm{s}}$ parameters are displayed in Table I. A small tail in the low-frequency domain makes the $\mathrm{CPE}_{\mathrm{s}}$ refinement difficult. A simple model consisting of the three elements was considered for the refinement of the EI spectra at $316 \mathrm{~K}$. However, by lowering the temperature, the refinement requires more than one time constant (Fig. 1(a)), and up to three $R / \mathrm{CPE}$ were then used (not shown). This is in agreement with previous results where three constant times $\mathrm{pF}, \mathrm{nF}$, and $\mu \mathrm{F}$ were reported to refine the shape of Nyquist plots. $^{30}$

At $316 \mathrm{~K}$, the values of the conductivity range from $10^{-9}$ to $10^{-10} \mathrm{~S} / \mathrm{cm}$. This is consistent with previous data reported for unhydrated cement ${ }^{25,39}$ and much lower than that reported for freshly hydrated cement paste. Indeed with cement paste dehydration, the continuous paths of water are found to break up and the conductance decreases steeply by at least five orders of magnitude. ${ }^{40}$ There is no trend in the conductivity value in both $\mathrm{C}-\mathrm{S}-\mathrm{H}$ and $\mathrm{C}-\mathrm{A}-\mathrm{S}-\mathrm{H}$ series. $\mathrm{CPE}_{\mathrm{p}}$ values range between $1.10^{-10}$ and $1.10^{-9} \Omega \cdot(\mathrm{rd} / \mathrm{s})_{\mathrm{p}}^{-n}$, and are in association with time constants between 1 and $15 \mathrm{~ms}$ (Table I). This is evidently much larger than those obtained on wet cement paste systems where time constants of the order of $\mu$ s are observed. ${ }^{30}$ This is in relation with the dry feature of both present series $\mathrm{C}-\mathrm{S}-\mathrm{H}$ and $\mathrm{C}-\mathrm{A}-\mathrm{S}-\mathrm{H}$. For the low-frequency response, $\mathrm{CPE}_{\mathrm{s}}$ values range between $10^{7}$ and $10^{8} \Omega \cdot(\mathrm{rd} / \mathrm{s})_{\mathrm{s}}^{-n}$, and the time constants between 0.1 and $1 \mathrm{~s}$ (Table I). Again, this is much greater than the usual ms expected for the double layer process.

With an increase in temperature up to $316 \mathrm{~K}$, a progressive displacement of the complex curve toward the origin together with a reduction in arc radii was observed (Fig. 1(a)). It means that by increasing the temperature, the conductivity of the samples increases steadily up to $316 \mathrm{~K}$. However it remains constant or even decreases above this temperature (Fig. 3). This indicates that the temperature is of great importance for the charge carriers' mobility. Such an increase in the 253-316 $\mathrm{K}$ temperature range is interpreted by a greater mobility while the motion of the charge carriers is strongly hindered above $316 \mathrm{~K}$. The decrease in conductivity can be interpreted by a progressive loss of water molecules. We surmise that the loss of interfacial water molecules in the vicinity of the grain boundaries even in small number may have a great effect on the disruption of the conductive paths. The temperature of occurrence is surprisingly low in comparison to the thermal behavior of the samples, even if one notes the ignition of weight loss above room temperature. ${ }^{33}$ Thus, the interstitial medium within the dry samples is found to be highly sensitive to an increase in temperature, because its effect is observed at a temperature slightly above room temperature. It is in agreement with previous data reported for Portland cement mortar where, at a temperature excess of $50^{\circ} \mathrm{C}$, sample impedance was found to be almost independent of temperature. ${ }^{41}$ Impedance response above $316 \mathrm{~K}$ will not be further considered. 

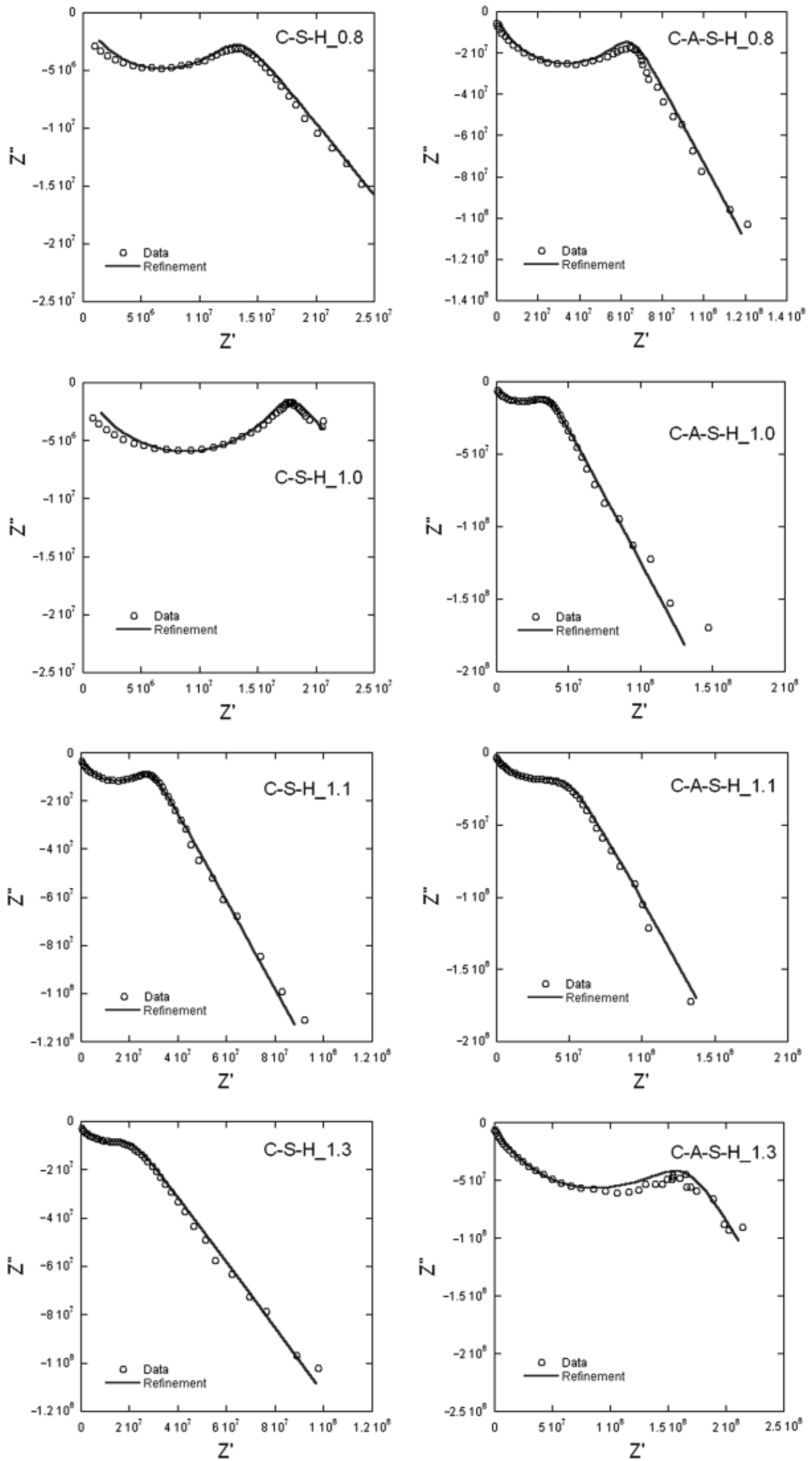

Fig. 2. Refinement of the Nyquist response adopting a three-element model $+\mathrm{CPE}_{\mathrm{s}} / \mathrm{CPE}_{\mathrm{p}} R$ at $316 \mathrm{~K}$ for $\mathrm{C}-\mathrm{S}-\mathrm{H}$ (left) and $\mathrm{C}-\mathrm{A}-\mathrm{S}-\mathrm{H}$ (right) series. Top to bottom from $0.8,1.0,1.1$ to 1.3 for the $\mathrm{C} / \mathrm{S}$ ratio. 


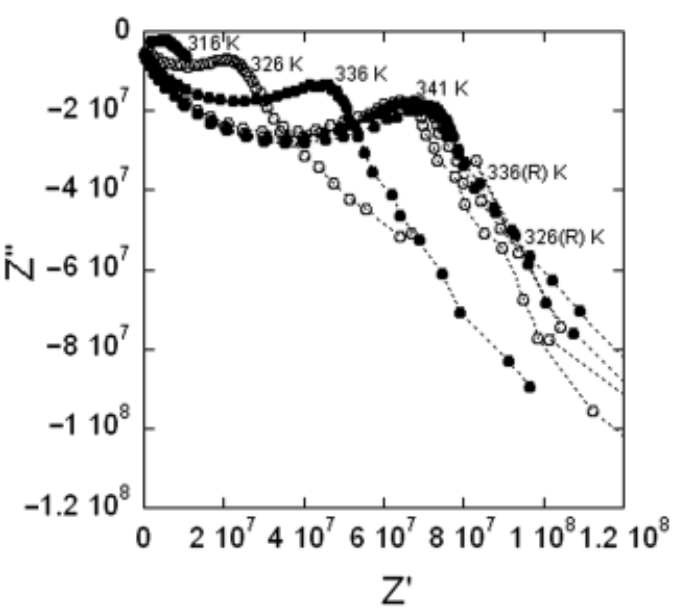

Fig. 3. Variation of Nyquist curves for C-S-H_1.1 above $316 \mathrm{~K} .(R)$ is used when the temperature cycle is reverse.

\section{(2) Fractal Surface}

The depression angle $\theta$ visualized by the depleted semicircle at high frequency is related to the exponent $\mathrm{n}$ also called the arc depression factor by the relation:

$$
n=1-(2 \theta / \pi)
$$

This parameter was linked to the fractal dimension of the gel surface and pore size distribution within the cement paste by the empirical relation $^{42}$ :

$$
n \approx\left(D_{\mathrm{s}}-1\right) / 2
$$

where $D_{\mathrm{s}}$ is the fractal dimension of the interface. Adopting (5), the values obtained from measurements were comparable to fractal BET theory as well as the neutron scattering study of hydrated cement paste. ${ }^{42,43}$ By plotting the arc depression factor versus hydration time, a constant value of $0.75-0.8$ was obtained after a sufficiently long time, suggesting then a characteristic value for $\mathrm{C}-\mathrm{S}-\mathrm{H} .{ }^{25}$ Similarly, the fractal geometry is used here to explain the depletion of the capacitive arc and the values are reported in Table I. Values around 0.7 are obtained for $n_{\mathrm{p}}$ and related to a fractal dimension of the pores surfaces ranging from 2.4 to 2.7 . This is slightly higher to what is reported for hydrated cement-based materials ${ }^{30}$ suggesting in our case a more ideal capacitive response. Indeed, $n_{\mathrm{p}}$ was related to the pore size distribution and its increase is usually associated with a reduction in the pore size and as well as a narrowing in the size distribution. It means that both $\mathrm{C}-\mathrm{S}-\mathrm{H}$ and $\mathrm{C}-\mathrm{A}-\mathrm{S}-\mathrm{H}$ series can be viewed as a continuous medium, the fractal dimension approaching the Euclidian dimension $\left(n_{\mathrm{p}}=1, D_{\mathrm{s}}=3\right)$.

\section{(3) Dielectric Study}

In dielectric representation, a time constant was assumed to correspond to the dielectric characteristics of the cement paste, and the associated capacitance value $\mathrm{C}$ should correspond to a parallel plate capacitor such as:

$$
C=\varepsilon \varepsilon_{0} / K_{\mathrm{c}}
$$

where $\varepsilon_{0}$ is the vacuum permittivity $\left(\varepsilon_{0}=8.85410^{-14} \mathrm{~F} / \mathrm{cm}\right), \varepsilon$ is the sample dielectric constant, and $K_{\mathrm{c}}$ the geometric cell constant. The apparent dielectric constant $\varepsilon$ is calculated to be $>100$ for both series. Evidently, this is not a true value due to the rather larger capacitance value of the order of $10^{-10} \mathrm{~F}$ instead of the expected $\mathrm{pF}$ order. It underlines that its origin is different from a bulk dielectric process alone; consequently, ionic motion should be considered in addition to grain boundaries dielectric polarization. In both $\mathrm{C}-\mathrm{S}-\mathrm{H}$ and $\mathrm{C}-\mathrm{A}-\mathrm{S}-\mathrm{H}$ series, the remaining water molecules are probably bounded in the interlayer of calcium silicate hydrate structure and they can no longer relax. This is in discrepancy with the change in conduction observed above room temperature that suggests rather weakly bounded water molecules. We surmise that the change in conduction may be attributed to the low amount of surface-adsorbed water molecules different from the strongly bounded water molecules that leaves the structure at a temperature up to $400^{\circ} \mathrm{C}$ as observed by the thermal analysis ${ }^{33}$ and in agreement with the literature. ${ }^{44}$

To scrutinize further the dielectric behavior that is influenced by various parameters such as porosity, water content, ion concentration and pore geometry and the behavior of the ions at the pore surface, the modulus loss factor formalism is adopted (see Section II). The representative plots of the modulus loss factor $M^{\prime \prime}$ as a function of frequency show Lorentzian peaks that shift toward higher frequency with an increase in temperature up to $316 \mathrm{~K}$ (Fig. 4). The shift in $M^{\prime \prime}$ peak maximum is indicative of transition from long to shorter range mobility of charge carriers. As for the conductivity, the shift of the peak is reversed when the temperature is $>316 \mathrm{~K}$ (not shown).

In the present set of data, the interfacial relaxation process is evident in all systems. The intensity of this relaxation increases in temperature; this becomes apparent from the increase of the dissipation factor (shown for $\mathrm{C}-\mathrm{A}-\mathrm{S}-\mathrm{H} \_0.8$ in Fig. 5) and from the reduction of the modulus loss factor (Fig. 4). It may be interpreted by a more pronounced interface. Moreover, the presence of the peaks is typical of Debye-type relaxation, ${ }^{45}$ even if the presence of a depressed semicircle on Nyquist plot (Figs. 1 and 3) suggests some deviation from the ideal Debye case. High values in the low-frequency range suggest the existence of one or more of the following processes: (i) electrode polarization, (ii) interfacial polarization Maxwell-Wagner-Sillars (MWS) effect, and (iii) conductivity phenomena. MWS effect occurs in systems exhibiting heterogeneity. On the other hand, the relaxation process recorded in the medium frequency region is attributed to interfacial polarization because electrode polarization is neglected in the electric modulus formalism ${ }^{38}$ and the conductivity of the samples is low (in the order of $1.10^{-10} \mathrm{~S} / \mathrm{cm}$ ). Its occurrence was ascribed to ionic polarization at the grains interface. When lowering the temperature, an asymmetric peak broadening occurs. This is indicative of a spread in the relaxation time.

The relaxation times were determined from the imaginary part of the modulus loss factor because it is model free and it may represent the most probable relaxation time. ${ }^{46,47}$

A peak is observed when the condition $\omega \tau=1$ is satisfied, where $\omega$ is the angular frequency and $\tau$ is the Debye relaxation time. Relaxation times $\tau$ were determined at the frequency corresponding to the peak maximum. Similar observations may be carried out on the dissipation factor $\tan \delta\left(\varepsilon^{\prime \prime} / \varepsilon^{\prime}\right)$ as the emergence of a peak or a shoulder is also the manifestation of interfacial polarization that is characteristic of heterogeneous systems and is caused by charges blocked at the internal phase boundaries. However, $\tan \delta$ variation does not exhibit a clear defined relaxation peak over the entire domain in temperature (Fig. 5), explaining the reason to consider $M^{\prime \prime}$. Relaxation times $\tau$ present a linear dependence against the inverse of temperature (Fig. 6). The activation energy of the relaxation is then calculated based on the following Arrhenius-type equation (Table II):

$$
\tau=\tau_{0} \exp \left(E_{\mathrm{a}} / k T\right)
$$

On a time scale, it can be physically interpreted with the time involved for the sample to reorganize structurally. Shorter relaxation times are indicative of faster dynamics at the interfaces. The calculated values of the preexponential factor $\tau_{0}$ can be rationalized between samples. 

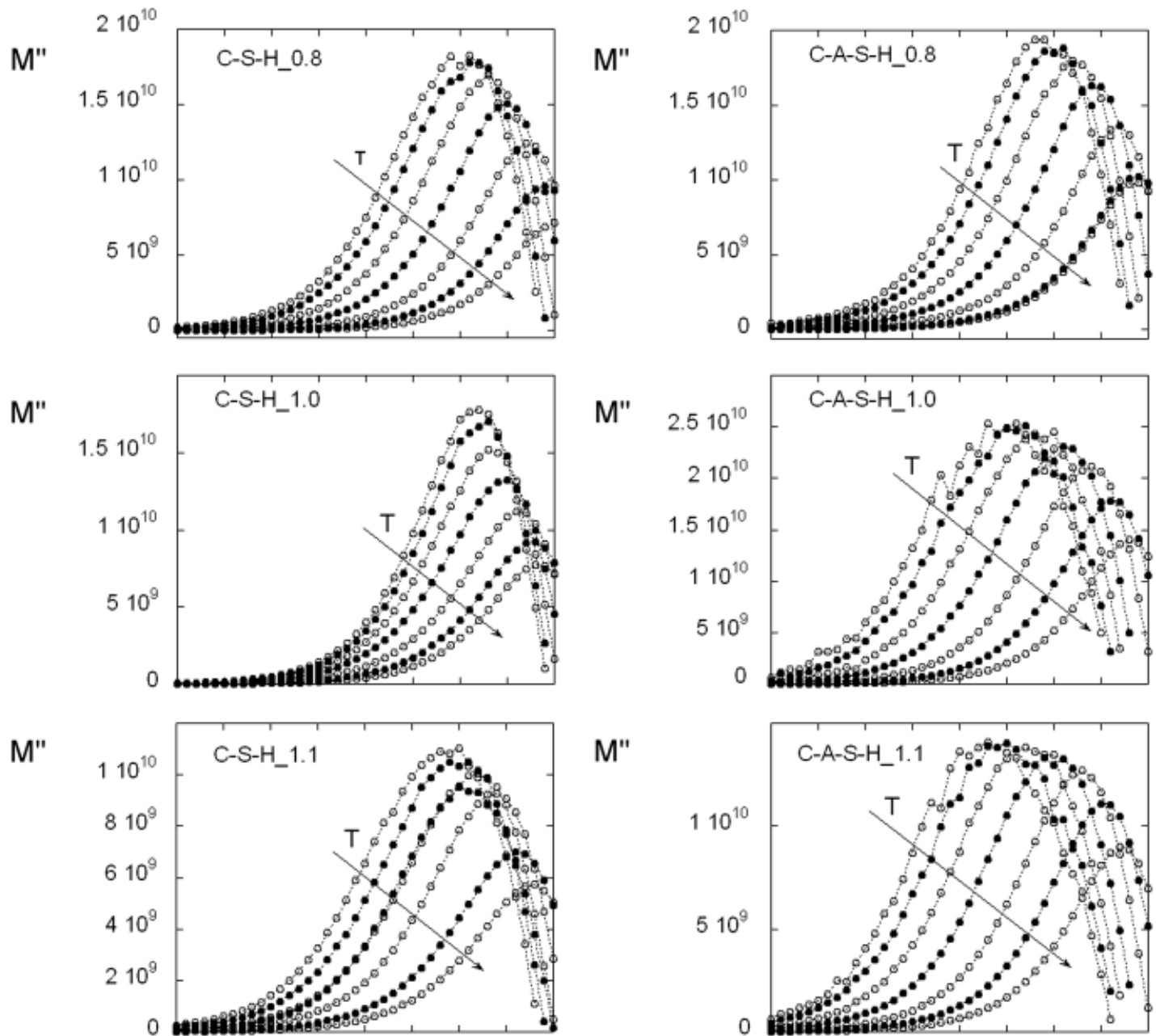

$M^{\prime \prime}$
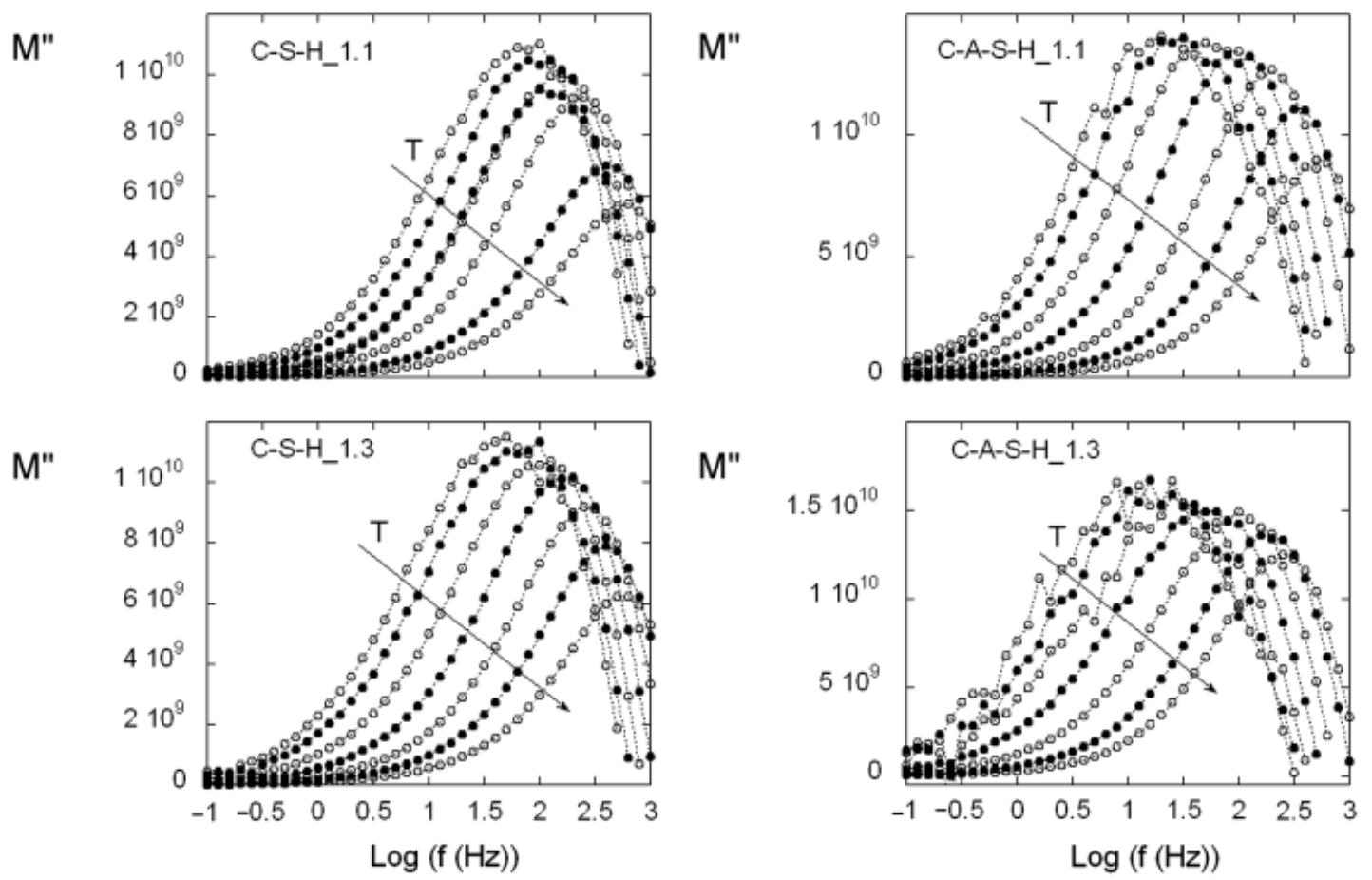

Fig. 4. Variation of the electric loss modulus $M^{\prime \prime}$ vs frequency over the 246-296 K temperature range. The arrows indicate the increase of the temperature.

The activation energy of $\mathrm{C}-\mathrm{S}-\mathrm{H}$ series ranges between 0.24 and $0.38 \mathrm{eV}$ while the one for $\mathrm{C}-\mathrm{A}-\mathrm{S}-\mathrm{H}$ ranges between 0.39 and $0.50 \mathrm{eV}$. Within each series, there is no pronounced trend for the activation energy against the ratio $\mathrm{Ca} / \mathrm{Si}$. The values are close to the other reported in the case of frozen fresh cement paste where an activation energy of $0.31 \mathrm{eV}$ was reported. ${ }^{48}$ Evidently, our values are higher (i.e., more temperature dependent) than that reported for ordinary Portland cement (OPC) pastes. ${ }^{41,49}$ However it is difficult to access to structural information and/or make a comparison with OPC because the activation energy is not only characteristic of the composition and phase ordering but also representative of grain boundaries, granulometry, and phase compactness.
The variation of $M^{\prime \prime}$ above $316 \mathrm{~K}$ (and in the cooling cycle), shown in Fig. 7 for $\mathrm{C}-\mathrm{A}-\mathrm{S}-\mathrm{H} \_$0.8, is found to be erratic as for $Z^{\prime \prime}$ (Fig. 3).

The plot of $E_{\mathrm{a}}$ and $\tau_{0}$ vs chemical composition provides some general trend (Fig. 8). Regardless of the chemical composition, the series $\mathrm{C}-\mathrm{A}-\mathrm{S}-\mathrm{H}$ exhibits greater activation energy than the series $\mathrm{C}-\mathrm{S}-\mathrm{H}$. It means that the process is more temperature dependent in samples $\mathrm{C}-\mathrm{A}-\mathrm{S}-\mathrm{H}$. Such an increase in mobility of the charge carriers between $\mathrm{C}-\mathrm{A}-\mathrm{S}-\mathrm{H}$ and $\mathrm{C}-\mathrm{S}-\mathrm{H}$ series can be explained by the increase of layer spacing of about $2 \AA$, corresponding to a more open structure, by the decrease of the coherent domain size, and/or by the presence of aluminum cations into the interlayer space as demonstrated previously. ${ }^{33,34}$ 


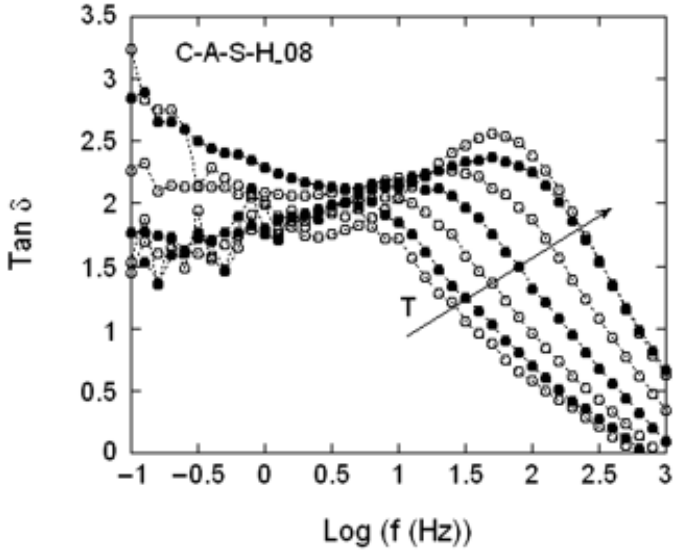

Fig. 5. Variation of the dissipation factor $\tan \delta$ vs frequency for $\mathrm{C}-\mathrm{A}-$ S-H_08 over the 253-316 K temperature range.

\section{Conclusion}

We demonstrate here that EIS is a suitable technique for the study of diffusion of charge carriers in disordered media such as $\mathrm{C}-\mathrm{S}-\mathrm{H}$ and $\mathrm{C}-\mathrm{A}-\mathrm{S}-\mathrm{H}$ powdered samples in spite of the rather small conductivity. By adopting a simple model using two CPE elements and one resistance, it was possible to refine the Nyquist curves for both $\mathrm{C}-\mathrm{S}-\mathrm{H}$ and $\mathrm{C}-\mathrm{A}-\mathrm{S}-\mathrm{H}$ series at $316 \mathrm{~K}$. Beyond this temperature, the conduction process was found to behave erratically (certainly due to the beginning of dehydration of sorbed water molecules) and was then not further considered.

The empirical relation between arc depression factor and the fractal structure was applied here. Its value ranges between 2.4 and 2.7 suggesting a rather homogeneous and dense bulk with
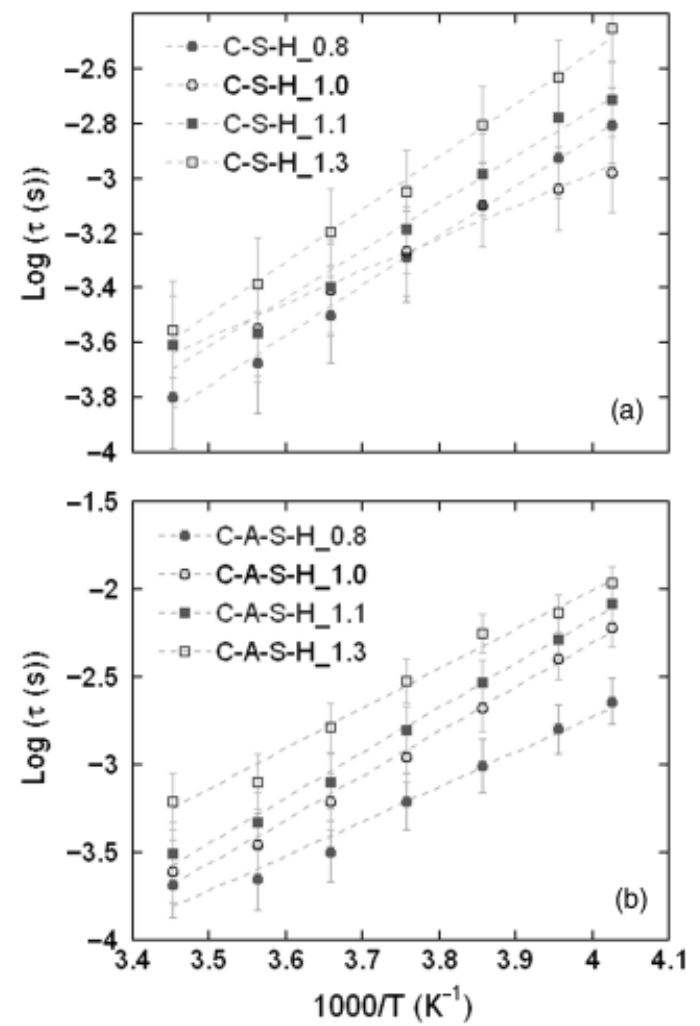

Fig. 6. Relaxation time $\tau$ issued from $\omega \tau=1$ position on $M^{\prime \prime}$ maximum peak over 246-296 K temperature range. The gray dashed lines visualized the linear fit.
Table II. Activation Energy $\boldsymbol{E}_{\mathrm{a}}$ and Preexponential Factor $\tau_{0}$ Obtained from $\omega \tau=1$ Position on $M^{\prime \prime}$ Maximum Peak over 253-316 K Temperature Range

\begin{tabular}{lcccc}
\hline & C-S-H_0.8 & C-S-H_1.0 & C-S-H_1.1 & C-S-H_1.3 \\
\hline$E_{\mathrm{a}}(\mathrm{eV})$ & 0.35 & 0.24 & 0.34 & 0.38 \\
$(\mathrm{~kJ} / \mathrm{mol})$ & $(33.7)$ & $(23.1)$ & $(32.7)$ & $(36.6)$ \\
$\log \tau_{0}$ & -10.0 & -7.7 & -9.6 & -10.2 \\
\hline
\end{tabular}

\begin{tabular}{lcccc} 
& C-A-S-H_0.8 & C-A-S-H_1.0 & C-A-S-H_1.1 & C-A-S-H_1.3 \\
\hline$E_{\mathrm{a}}(\mathrm{eV})$ & 0.39 & 0.50 & 0.50 & 0.45 \\
$(\mathrm{~kJ} / \mathrm{mol})$ & $(37.6)$ & $(48.2)$ & $(48.2)$ & $(43.3)$ \\
$\log \tau_{0}$ & -10.6 & -12.3 & -12.4 & -11.1 \\
\hline
\end{tabular}

no extended grain boundaries such as observed in the case of hydrated cement-type materials. The $\mathrm{CPE}_{\mathrm{s}}$ element responsible of the low-frequency response presents a rather small $n_{\mathrm{s}}$ value, thus showing an extended roughness of the electrode to sample interface that was not further optimized. The electric lost modulus $M^{\prime \prime}$ is found to be highly sensitive to the temperature change. Studied in the temperature range from 253 to $316 \mathrm{~K}$, its variation is found to follow Arrhenius dependence with activation energy slightly higher for $\mathrm{C}-\mathrm{A}-\mathrm{S}-\mathrm{H}$ than for $\mathrm{C}-\mathrm{S}-\mathrm{H}$ series, which was related to the different structures of the two kinds of samples.

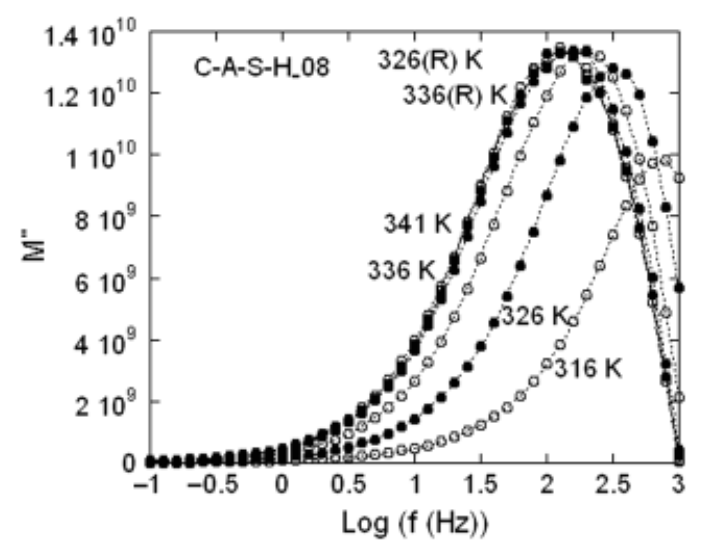

Fig. 7. Variation of the electric loss modulus $M^{\prime \prime}$ for C-A-S-H_08 vs frequency above $316 \mathrm{~K} .(R)$ is used when the temperature cycle is reverse.

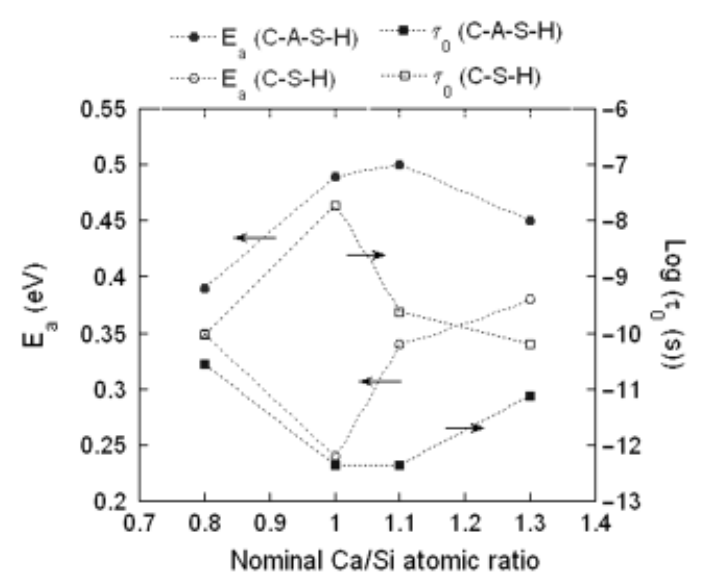

Fig. 8. Variation of the activation energy $E_{\mathrm{a}}$ and preexponential factor $\tau_{0}$ as a function of $\mathrm{Ca} / \mathrm{Si}$ nominal chemical composition. The arrows report to the $y$-axis reading. 


\section{References}

${ }^{1}$ H. F. W. Taylor, "Hydration of Portland cement"; pp. 187-226 in Cement Chemistry. Chapter 7, 2nd edition, Edited by T. Telford. Thomas Telford Publishing, London, 1997.

${ }^{2}$ M. D. Andersen, H. J. Jakobsen, and J. Skibsted, "Incorporation of Aluminum in the Calcium Silicate Hydrate (C-S-H) of Hydrated Portland Cements: A HighField ${ }^{27} \mathrm{Al}$ and ${ }^{29} \mathrm{Si}$ MAS NMR Investigation," Inorg. Chem., 42 [7] 2280-7 (2003).

${ }^{3}$ I. G. Richardson, A. R. Brough, R. Brydson, G. W. Groves, and C. M. Dobson, "Location of Aluminum in Substituted Calcium Silicate Hydrate (C-S-H) Gels as Determined by ${ }^{29} \mathrm{Si}$ and ${ }^{27} \mathrm{Al}$ NMR and EELS," J. Am. Ceram. Soc., 76 [9] 2285-8 (1993).

${ }^{4}$ M. D. Andersen, H. J. Jakobsen, and J. Skibsted, "Characterization of White Portland Cement Hydration and the C-S-H Structure in the Presence of Sodium Aluminate by ${ }^{27} \mathrm{Al}$ and ${ }^{29} \mathrm{Si}$ MAS NMR Spectroscopy," Cem. Concr. Res., 34 [5] $857-68(2004)$

${ }^{5}$ P. Faucon, A. Delagrave, J. C. Petit, C. Richet, J. M. Marchand, and H. Zanni, "Aluminum Incorporation in Calcium Silicate Hydrates (C-S-H) Depending on their Ca/Si Ratio," J. Phys. Chem. B, 103 [37] 7796-802 (1999).

${ }^{6}$ G. K. Sun, J. F. Young, and R. J. Kirkpatrick, "The Role of Al in C-S-H: NMR, XRD, and Compositional Results for Precipitated Samples," Cem. Concr. Res., 36 [1] 18-29 (2006).

${ }^{7}$ X. Pardal, I. Pochard, and A. Nonat, "Experimental Study of Si-Al Substitution in Calcium-Silicate-Hydrate (C-S-H) Prepared Under Equilibrium Conditions," Cem. Concr. Res., 39 [8] 637-43 (2009).

${ }^{8} \mathrm{H}$. M. Jennings, "Refinements to Colloid Model of CSH in Cement: CM-II," Cem. Concr. Res., 38 [3] 275-89 (2008).

${ }^{9}$ P. R. Camp and S. Bilotta, "Dielectric Properties of Portland Cement Paste as a Function of Time Since Mixing," J. Appl. Phys., 66 [12] 6007-13 (1989).

${ }^{10} \mathrm{P}$. Gu and J. J. Beaudoin, "Dielectric Behavior of Hardened Cement Paste Systems," J. Mater. Sci. Lett., 15 [2] 182-4 (1996).

${ }^{11}$ D. Buerchler, B. Elsener, and H. Boehni, "Electrical Resistivity and Dielectric Properties of Hardened Cement Paste and Mortar," Mater. Res. Soc. Symp. Proc., 411, 407-12 (1996).

${ }^{12}$ P. Gu, P. Xie, J. J. Beaudoin, and R. Brousseau, "A.C. Impedance Spectroscopy (II): Microstructural Characterization of Hydrating Cement-Silica Fume Systems," Cem. Concr. Res., 23 [1] 157-68 (1993).

${ }^{13}$ W. J. McCarter, G. Starrs, and T. M. Chrisp, "The Complex Impedance Response of Fly-Ash Cements Revisited," Cem. Concr. Res., 34 [10] 1837-43 (2004)

${ }^{14}$ F. D. Tamas, E. Farkas, M. Voros, and D. M. Roy, "Low-Frequency Electrical Conductivity of Cement, Clinker and Clinker Mineral Pastes," Cem. Concr. Res., 17 [2] 340-8 (1987).

${ }^{15}$ G. Levita, A. Marchetti, G. Gallone, A. Princigallo, and G. L. Guerrini, "Electrical Properties of Fluidified Portland Cement Mixes in the Early Stage of Hydration," Cem. Concr. Res., 30 [6] 923-30 (2000).

${ }^{16}$ M. Moukwa, M. Brodwin, S. Christo, J. Chang, and S. P. Shah, "The Influence of the Hydration Process Upon Microwave Properties of Cements," Cem Concr. Res., 21 [5] 863-72 (1991).

${ }^{17}$ J. M. Torrents, J. Roncero, and R. Gettu, "Utilization of Impedance Spectroscopy for Studying the Retarding Effect of a Superplasticizer on the Setting of Cement," Cem. Concr. Res., 28 [9] 1325-33 (1998).

${ }^{18}$ T. O. Mason, M. A. Campo, A. D. Hixon, and L. Y. Woo, "Impedance Spectroscopy of Fiber-Reinforced Cement Composites," Cem. Concr. Compos., 24 [5] 457-66 (2002)

${ }^{19}$ B. Jönsson, H. Wennerström, A. Nonat, and B. Cabane, "Onset of Cohesion in Cement Paste," Langmuir, 20 [16] 6702-9 (2004).

${ }^{20}$ L. Nachbaur, J. C. Mutin, A. Nonat, and L. Choplin, "Dynamic Mode Rheology of Cement and Tricalcium Silicate Pastes from Mixing to Setting," Cem. Concr. Res., 31 [2] 183-92 (2001).

${ }^{21} \mathrm{C}$. Labbez, B. Jönsson, I. Pochard, A. Nonat, and B. Cabane, "Surface Charge Density and Electrokinetic Potential of Highly Charged Minerals: Experiments and Monte Carlo Simulations on Calcium Silicate Hydrate," J. Phys. Chem. B, 110 [18] 9219-30 (2006).

${ }^{22}$ C. Labbez, A. Nonat, I. Pochard, and B. Jönsson, "Experimental and Theoretical Evidence of Overcharging of Calcium Silicate Hydrate," J. Colloid Interface Sci., 309 [2] 303-7 (2007).

${ }^{23}$ C. Labbez, B. Jönsson, M. Skarba, and M. Borkovec, "Ion-Ion Correlation and Charge Reversal at Titrating Solid Interfaces," Langmuir, 25 [13] 7209-13 (2009).

${ }^{24}$ M. Keddam, H. Takenouti, X. R. Novoa, and C. Andrade, "Impedance Measurements on Cement Paste," Cem. Conc. Res., 27 [8] 1191-201 (1997).

${ }^{25}$ B. J. Christensen, R. T. Coverdale, R. A. Olson, S. J. Ford, E. J. Garboczi, H. M. Jennings, and T. O. Mason, "Impedance Spectroscopy of Hydrating Cement-Based Materials: Measurement, Interpretation, and Application," J. Am. Ceram. Soc., 77 [11] 2789-804 (1994).
${ }^{26}$ B. J. Christensen, T. O. Mason, and H. M. Jennings, "Influence of Silica Fume on the Early Hydration of Portland Cements Using Impedance Spectroscopy," J. Am. Ceram. Soc., 75 [4] 939-45 (1992).

${ }^{27}$ P. Gu, P. Xie, Y. Fu, and J. J. Beaudoin, "A.C Impedance Phenomena in Hydrating Cement Systems: Frequency Dispersion Angle and Pore Size Distribution," Cem. Concr. Res., 24 [1] 86-8 (1994).

${ }^{28}$ R. T. Coverdale, B. J. Christensen, T. O. Mason, H. M. Jennings, and E. J. Garboczi, "Interpretation of the Impedance Spectroscopy of Cement Paste via Computer Modelling, Part II Dielectric Response," J. Mater. Sci., 29 [19] 4984-92 (1994). $\left.{ }^{29} \mathrm{C}\right)$

${ }^{29} \mathrm{C}$. Andrade, V. M. Blanco, A. Collazo, M. Keddam, X. R. Novoa, and H. Takenouti, "Cement Paste Hardening Process Studied by Impedance Spectroscopy," Electrochim. Acta, 44 [24] 4313-8 (1999).

${ }^{30}$ C. Alonso, C. Andrade, M. Keddam, X. R. Novoa, and H. Takenouti, "Study of the Dielectric Characteristics of Cement Paste," Mater. Sci. Forum, 289-292, $15-28(1998)$

${ }^{31}$ Y. El Hafiane, A. Smith, J. P. Bonnet, P. Abélard, and P. Blanchart, "Electrical Characterization of Aluminous Cement at the Early Age in the $10 \mathrm{~Hz}-1 \mathrm{GHz}$ Frequency Range," Cem. Conc. Res., 30 [7] 1057-62 (2000).

${ }^{32}$ L. Y. Woo, N. J. Kidner, S. Wansom, and T. O. Mason, "Combined Time Domain Reflectometry and AC-Impedance Spectroscopy of Fiber-Reinforced Fresh-Cement Composites," Cem. Concr. Res., 37 [1] 89-95 (2007).

${ }^{33}$ G. Renaudin, J. Russias, F. Leroux, F. Frizon, and C. Cau-dit-Coumes, "Structural Characterization of C-S-H and C-A-S-H Samples-Part I: LongRange Order Investigated by Rietveld Analyses," J. Solid State Chem., 182 [12] 3312-9 (2009).

${ }^{34}$ G. Renaudin, J. Russias, F. Leroux, C. Cau-dit-Coumes, and F. Frizon, "Structural Characterization of C-S-H and C-A-S-H Samples-Part II: Local Environment Investigated by Spectroscopic Analyses," J. Solid State Chem., 182 [12] 3320-9 (2009).

${ }^{35}$ M. Cabeza, M. Keddam, X. R. Novoa, I. Sanchez, and H. Takenouti, "Impedance Spectroscopy to Characterize the Pore Structure During the Hardening Process of Portland Cement Paste," Electrochim. Acta, 51 [8-9] 1831-41 (2006).

${ }^{36}$ M. Cabeza, P. Merino, A. Miranda, X. R. Novoa, and I. Sanchez, "Impedance Spectroscopy Study of Hardened Portland Cement Paste," Cem. Concr. Res., 32 [6] 881-91 (2002).

${ }^{37}$ K. G. Gatos, J. G. Martinez Alcazar, G. C. Psarras, R. Thomann, and J. Karger-Kocsis, "Polyurethane Latex/Water Dispersible Boehmite Alumina Nanocomposites: Thermal, Mechanical and Dielectrical Properties," Comp. Sci. Technol., 67 [2] 157-67 (2007).

${ }^{38} \mathrm{G}$. M. Tsangaris, G. C. Psarras, and N. Kouloumbi, "Electric Modulus and Interfacial Polarization in Composite Polymeric Systems," J. Mater. Sci., 33 [8] 2027-37 (1998)

${ }^{39}$ D. E. Wilkosz and J. F. Young, "Effect of Moisture Adsorption on the Electrical Properties of Hardened Portland Cement Compacts," J. Am. Ceram. Soc., 78 [6] 1673-9 (1995)

${ }^{40}$ A. Berg, G. A. Niklasson, K. Brantervik, B. Hedberg, and L. O. Nilsson, "Dielectric Properties of Cement Mortar as a Function of Water Content," J. Appl. Phys., 71 [12] 5897-903 (1992).

${ }^{41}$ W. J. McCarter, "Effects of Temperature on Conduction and Polarization in Portland Cement Mortar," J. Am. Ceram. Soc., 78 [2] 411-5 (1995).

${ }^{42}$ G. A. Niklasson, A. Berg, K. Brantervik, B. Hedberg, and L. O. Nilsson, "Dielectric Properties of Porous Cement Mortar: Fractal Surface Effects," Solid State Commun., 79 [1] 93-6 (1991).

${ }^{43}$ R. A. Livingston, "Fractal Nucleation and Growth Model for the Hydration of Tricalcium Silicate," Cem. Concr. Res., 30 [12] 1853-60 (2000).

${ }^{44}$ K. Garbev, M. Bornefeld, G. Beuchle, and P. Stemmermann, "Cell Dimensions and Composition of Nanocrystalline Calcium Silicate Hydrate Solid Solutions. Part 2: X-Ray and Thermogravimetry Study," J. Am. Ceram. Soc., 91 [9] 3015-23 (2008).

${ }^{45}$ H. C. Kim and S. S. Yoon, "Dynamic Dielectric Analysis During Early-Stage Hydration of Ordinary Portland Cement," J. Phys. D: Appl. Phys., 21 [7] 1215-20 (1988).

${ }^{46}$ N. Shinyashiki, D. Imoto, and S. Yagihara, "Broadband Dielectric Study of Dynamics of Polymer and Solvent in Poly (vinyl pyrrolidone)/Normal Alcohol Mixtures," J. Phys. Chem. B, 111 [9] 2181-7 (2007).

${ }^{47}$ J. Mijovic, S. Ristic, and J. Kenny, "Dynamics of Six Generations of PAMAM Dendrimers As Studied by Dielectric Relaxation Spectroscopy," Macromolecules, 40 [14] 5212-21 (2007).

${ }^{48}$ S. S. Yoon, S. Y. Kim, and H. C. Kim, "Dielectric Spectra of Fresh Cement Paste Below Freezing Point Using an Insulated Electrode," J. Mater. Sci., 29 [7] 1910-4 (1994)

${ }^{49}$ A. Atkinson and A. K. Nickerson, "The Diffusion of Ions Through WaterSaturated Cement," J. Mater. Sci., 19 [9] 3068-78 (1984). 